\title{
Talin depletion reveals independence of initial cell spreading from integrin activation and traction
}

\author{
Xian Zhang ${ }^{1}$, Guoying Jiang ${ }^{1}$, Yunfei Cai ${ }^{1}$, Susan J. Monkley ${ }^{2}$, David R. Critchley $^{2}$, and \\ Michael P. Sheetz ${ }^{1,3}$ \\ ${ }^{1}$ Department of Biological Sciences, Columbia University, 1212 Amsterdam Avenue, New York, \\ New York 10027, USA \\ 2 Department of Biochemistry, University of Leicester, University Road, Leicester LE1 9HN, UK
}

\section{Abstract}

Cell spreading, adhesion and remodelling of the extracellular matrix (eCM) involve bi-directional signalling and physical linkages between the eCM, integrins and the cell cytoskeleton ${ }^{1-3}$. the actinbinding proteins talin 1 and 2 link ligand-bound integrins to the actin cytoskeleton and increase the affinity of integrin for the $\mathrm{eCM}^{4-6}$. Here we report that depletion of talin2 in talin1-null (talin1 ${ }^{-1-}$ ) cells did not affect the initiation of matrix-activated spreading or src family kinase ( $\mathrm{sFK}$ ) activation, but abolished the eCM-integrin-cytoskeleton linkage and sustained cell spreading and adhesion. specifically, focal adhesion assembly, focal adhesion kinase (FAK) signalling and traction force generation on substrates were severely affected. the talin 1 head domain restored $\beta 1$ integrin activation but only full-length talin1 restored the eCM-cytoskeleton linkage and normal cytoskeleton organization. Our results demonstrate three biochemically distinct steps in fibronectin-activated cell spreading and adhesion: 1) fibronectin-integrin binding and initiation of spreading, 2) fast cell spreading and 3) focal adhesion formation and substrate traction. We suggest that talin is not required for initial cell spreading. However, talin provides the important mechanical linkage between ligandbound integrins and the actin cytoskeleton required to catalyse focal adhesion-dependent pathways.

Disruption of the talinl gene in undifferentiated embryonic stem (ES) cells severely disrupts cell adhesion and cytoskeleton organization ${ }^{7}$. However, fibroblasts derived from talin $1^{-1-}$ ES cells spread and adhere normally, presumably due to increased expression of the closely related talin2 $\left(\mathrm{refs}^{7,8}\right)$. To study the role of talin in initial cell responses to ECM signals, we constructed a mouse talin 2 short interfering RNA (siRNA) expression plasmid and transfected it into the talin $1^{-1-}$ fibroblasts. We observed that talin 2 siRNA-expressing talin $1^{-1-}$ cells began to round up in culture after $48 \mathrm{~h}$. After 3.5 days, $77 \pm 11 \%$ of the cells became rounded (mean \pm s.d., $n>450,3$ repetitions), whereas most of the control siRNA-expressing cells showed an elongated morphology in culture. Co-transfection of a GFP-talin1 plasmid rescued the morphology change induced by talin 2 siRNA $(11 \pm 3 \%$ and $15 \pm 8 \%$ rounded up in control siRNA transfected and GFP-talin1 and talin2 siRNA co-transfected cells, respectively; mean

\footnotetext{
3Correspondence should be addressed to M.P.S. (e-mail: ms2001@ columbia.edu).

Note: Supplementary Information is available on the Nature Cell Biology website.

AUTHOR CONTRIBUTIONS

X.Z. performed all the experiments in M.P.S. lab with significant support from G.J. and Y.C.; S.J.M. provided the cell line and important information; D.R.C. provided important direction on the project.

COMPETING FINANCIAL INTERESTS

The authors declare no competing financial interests.

Published online at http://www.nature.com/naturecellbiology/

Reprints and permissions information is available online at http://npg.nature.com/reprintsandpermissions/
} 
\pm s.d., $n>450,3$ repetitions; Supplementary Information, Fig. S1a, b). Immunoblot analyses with a pantalin antibody $8 \mathrm{~d} 4$ and a talin2-specific antibody showed a significant reduction in talin2 expression 3.5 days after talin 2 siRNA transfection, whereas the levels of vinculin and $\beta 1$ integrin were not affected (Fig. 1b). Morphology of a wild-type mouse embryonic fibroblast, which expressed talin 1 but no detectable talin2, was not affected by talin 2 siRNA (data not shown). Thus, talin 2 is responsible for the morphology adopted by talin $1^{-1-}$ cells.

To determine whether loss of talin would completely block cell spreading, we collected cells 3.5 days after siRNA transfection and plated them on fibronectin-coated coverslips for $90 \mathrm{~min}$ before fixation. Consistent with the observations in long-term culture, $\operatorname{talin} 1^{-1-}$ cells cotransfected with GFP and control siRNA or with GFP-talin1 and talin2 siRNA elongated normally ( $10 \pm 2 \%$ and $16 \pm 4 \%$, respectively, were rounded), whereas most of the GFP and talin 2 siRNA co-transfected cells were rounded ( $65 \pm 7 \%$ rounded; mean \pm s.d., $n>450,3$ repetitions; Fig. 1a; Supplementary Information, Fig. S1c). However, a distinct subpopulation of 'partially spread' talin-deficient cells showed a thick central region and motile active edge (Supplementary Information, Fig. S1e, arrow). Time-lapse microscopy revealed robust early edge extension in talin-deficient cells, but a large portion of the cells retracted their protrusions and rounded up at later times (43\% rounded up and $16 \%$ retained complete or partial edge protrusions within $90 \mathrm{~min} ; n=193$; Fig. 1d, e; Supplementary Information, Movie 2). Typically, talin-deficient cells rounded up 28 min (median) after initiation of spreading (Fig. 1f). In contrast, $85 \%$ of $\operatorname{talin}^{-/-}$control cells (which express talin2) showed early overall spreading activity followed by regional protrusions $(n=218$; Fig. 1c, e; Supplementary Information, Movie 1). Thus, initial cell spreading, which is triggered by integrin-fibronectin interactions (Supplementary Information, Fig. S2), does not require talin, although it is required for sustained cell spreading.

Talin acts as a scaffold for other focal adhesion proteins ${ }^{4}$. Both talin 1 and talin2 localized to focal adhesions in fully spread and elongated cells (Supplementary Information, Fig. S1d, f) and no vinculin foci were observed in the edge protrusions of spread talin-deficient cells. Instead, vinculin appeared diffuse in the cytoplasm (Fig. 2b, d), as did paxillin (Fig. 2f). Talin depletion caused marked changes in actin cytoarchitecture. Distinct arc-shaped actin bundles and focal adhesion-associated dorsal stress fibres were missing in talin-deficient cells ${ }^{9}$. Instead, diffuse phalloidin staining and radial actin cables were seen in the thin edge protrusions (Fig. $2 b$, f). In talin-deficient cells, focal adhesion localization of actin regulatory protein mouse ENA (Mena) was also disrupted, and Mena distributed exclusively at the tip of cell edge protrusions ${ }^{10}$ (Fig. 2 g, h). Consistent with the loss of focal adhesions, the ventral cell membrane was partially detached from the substrate, shown by the missing total internal reflection fluorescence (TIRF) signal of cytoplasmic GFP (Fig. 2i, j; Supplementary Information, Movie 3 ). Both the loss of focal adhesions and detachment of the cell membrane from the substrate indicate adhesion defects in spreading talin-deficient cells and explain the short lifespan of the spread state.

Binding of the talin head domain modulates the affinity of integrins for ECM and the talin rod domain also binds to integrins directly ${ }^{11-13}$. Neither GFP-talin1 head (talin1H) nor GFPtalin 1 rod (talin1R) rescued the talin2 knockdown phenotype in culture (Supplementary Information, Fig. S3a, c). However, GFP-talin $1 \mathrm{H}$-expressing cells were able to maintain the edge protrusions on fibronectin for more than $90 \mathrm{~min}$, whereas they failed to achieve the elongated morphology of control cells ('partially spread' cells in fixed samples increased to $43 \pm 12 \%$; mean \pm s.d., $n>400,3$ repetitions; Fig. 3a; Supplementary Information, Fig. S3d). Spreading of GFP-talin1R-expressing cells was no different from that of talin-deficient cells on fibronectin, and the cells rounded up before $90 \mathrm{~min}$ (Fig. 3a; Supplementary Information, Fig. S3d). Time-lapse microscopy revealed that $46 \%$ of talin $1 \mathrm{H}-$ expressing cells maintained stable edge protrusions for more than 90 min (Fig. 3b, c; Supplementary Information, Movie 
4). Immunostaining for vinculin and paxillin did not detect focal adhesions, indicating that talin1H-expressing cells were not stabilized by any remaining talin2 (Fig. 3d, e).

Although GFP-talin1H did not localize to discrete foci, it was concentrated at the leading edge of the cell protrusions (Fig. 3d, e, g). An explanation for the sustained spread state was that talin $1 \mathrm{H}$ increased the binding affinity of integrins for fibronectin. Indeed, integrin-activating $\mathrm{Mn}^{2+}$ increased spread-state duration of talin-deficient cells on fibronectin without rescuing focal adhesion formation (Supplementary Information, Fig. S3e-h, Movie 5). The 9EG7 antibody, which recognizes activated $\beta 1$ integrin $^{14,15}$, revealed colocalization of activated $\beta 1$ integrin with GFP-talin1H at the leading edge of talin1H-expressing cells (Fig. 3g) and with GFP-talin1 in talin1-restored cells (Fig. 3f). There was very little activated $\beta 1$ integrin staining in talin-deficient cells (Fig. 3h). This suggests that talin, through its head domain, specifically activates and binds to $\beta 1$ integrin, which localizes at the leading edge of the cell through highaffinity fibronectin binding ${ }^{15}$.

Both FAK and SFK are early mediators in ECM-activated cell spreading ${ }^{16-18}$. However, it is unclear whether their activities are dependent on talin. In talin $1^{-1-}$ control cells, the phosphorylation of FAK on Tyr 397 (which is involved in the initial FAK activation) rapidly increased after plating on fibronectin. In talin-deficient cells, a similar trend was seen but the level of Tyr 397 phosphorylation decreased in proportion to the magnitude of talin2 depletion (Fig. 4a, b). This indicates that talin is necessary for the normal response of FAK on adhesion to fibronectin. Although talin $1 \mathrm{H}$ associates with $\mathrm{FAK}^{19}$, re-expression of talin $1 \mathrm{H}$ did not rescue FAK-Tyr 397 phosphorylation on spreading (Fig. 4a, b). On the other hand, both control and talin-deficient cells showed a comparable increase in SFK activity 10 min after spreading (pTyr 416: Src level increased by more than twofold $10 \mathrm{~min}$ after spreading, compared with that in cells kept in suspension; Fig. 4a). In talin1H-expressing cells, the activity of SFK showed a slightly greater increase (Fig. 4a, c).

Phosphorylation of FAK-Tyr 397 in spreading control cells was dependent on myosin II contractility (Supplementary Information, Fig. S4), indicating the involvement of traction force in FAK activation ${ }^{20}$. The lack of FAK-Tyr 397 phosphorylation and the presence of accelerated actin rearward flow driven by myosin contractility in adhesion-defective cells (Fig. 5c-f) suggest that talin-dependent adhesions may provide the anchoring sites for substrate traction and FAK activation. Consistent with this view, phospho-FAK (pTyr 397) localized to the distal tip of focal adhesions and dorsal stress fibres in control cells (Fig. 4d) and there was very little specific phospho-FAK signal in the protrusions of talin-deficient and talin1H-expressing cells (Fig. 4e, f). Our observations (Fig. 4c; Supplementary Information, Fig. S4) also suggest that SFK activation upon spreading is independent of talin or cytoskeleton contraction ${ }^{3}$.

Formation of focal adhesion correlates with the slowing of myosin II-driven actin rearward flow at the back of lamellipodia ${ }^{21}$. We studied the actin dynamics using $\alpha$-actinin-GFP, which co-distributed with the actin cytoskeleton ${ }^{22}$ (Supplementary Information, Fig. S5a, b). In control cells, differential interference contrast (DIC) and TIRF microscopy revealed fast $\alpha$ actinin rearward flow $\left(50.3 \pm 8.2 \mathrm{~nm} \mathrm{~s}^{-1}\right)$ within the region $2.4 \pm 0.4 \mu \mathrm{m}$ from the active leading edge; flows were occasionally followed by the formation of immobilized $\alpha$-actinin foci (mean \pm s.d., $n=16$ cells; Fig. 5a, b; Supplementary Information, Movie 6). In contrast, talin1Hexpressing cells showed a marked increase in $\alpha$-actinin rearward flow-rate throughout the stable protrusions, observed by DIC, TIRF and epifluorescence microscopy $\left(118.7 \pm 15.4 \mathrm{~nm} \mathrm{~s}^{-1}\right.$, within the protrusion width of $6.4 \pm 1.0 \mu \mathrm{m}$, mean \pm s.d., $n=10$ cells; Fig. $5 \mathrm{c}$, d; Supplementary Information, Movie 7). No noticeable $\alpha$-actinin foci were formed on the substrate (Fig 5c,d). A comparable increase in rearward flow-rate was also observed in talin-deficient cells when they transiently retained their maximum spread area on fibronectin (flow-rate, $112.4 \pm 16.5$ $\mathrm{nm} \mathrm{s}^{-1}$, within the width of $6.5 \pm 0.8 \mu \mathrm{m}$, mean \pm s.d., $n=14$ cells). Furthermore, expression 
of an actin-binding mutant, talin $1^{\mathrm{ABS}}$ (ref. ${ }^{5}$ ), which lacks the carboxy-terminal actin-binding module, was unable to restore normal cytoskeleton organization and focal adhesion formation or to confine the fast rearward flow to the cell periphery (flow-rate, $109.8 \pm 11.2 \mathrm{~nm} \mathrm{~s}^{-1}$, within $6.2 \pm 0.7 \mu \mathrm{m}$ from the leading edge, mean \pm s.d., $n=14$ cells; Supplementary Information, Fig. $\mathrm{S} 5 \mathrm{c}-\mathrm{h})$.

We did not observe hyperactivation of myosin II with talin depletion (Supplementary Information, Fig. S6a, b). Inhibition of myosin II activity using blebbistatin significantly decreased the rate of actin rearward flow in talin $1 \mathrm{H}$-expressing cells (flow-rate decreased to $45.1 \pm 7.2 \mathrm{~nm} \mathrm{~s}^{-1}$, mean \pm s.d., $n=9$ cells; Fig. 5e, f; Supplementary Information, Movie 8). Shortly after treatment, the perinuclear boundary seen in DIC disappeared and gradual outward movement of previously restricted vesicles was observed. To understand how myosin II drives the rapid radial flow in the absence of adhesions, we stained phosphomyosin light chain (MLC) for the distribution of myosin II activity. In control (data not shown) and GFP-talin1 restored cells, most of the active myosin II localized to the fine circumferential actin filaments parallel to the lamellipodial edge and prominent actin arc bundles (Fig. $5 \mathrm{~g}$ ). Although the localization of phospho-MLC suggests that the main contraction was focused along the actin arcs, these structures moved rearward at a relatively low rate $\left(7.3 \pm 3.0 \mathrm{~nm} \mathrm{~s}^{-1}\right.$, mean \pm s.d., $n=15$ cells $)$, which may be explained by the hindrance resulting from their interaction with the dorsal stress fibres emanating from focal adhesions ${ }^{23}$ (Fig. 5g, inset). In talin-deficient (data not shown) and talin1H-expressing cells, where there were no focal adhesions or stress fibres, a diffuse phospho-MLC pattern was observed in edge protrusions and most of the phospho-MLC was in the perinuclear region, colocalizing with condensed circumferential actin (Fig. 5h). This suggests that a talin-cytoskeleton linkage is essential in slowing the rearward actin flow, probably by organizing and coupling the actomyosin contraction to the ECM.

Loss of a physical connection between the ECM and contractile cytoskeleton caused by talin depletion should lead to a defect in traction force generation on substrates. Observation of cell spreading on a fibronectin-coated elastic surface confirmed that traction force generation was significantly reduced in talin-deficient and talin $1 \mathrm{H}$-expressing cells ${ }^{24}$ (Supplementary Information, Fig. S6c-e, Movies 9, 10). Therefore, slowing of actin rearward flow, coupling of actomyosin contraction and force generation on the substrate correlate with the presence of full-length talin and focal adhesion formation.

Actin flow in peripheral regions can entrap and pull microtubules inwards ${ }^{25}$. In talin1Hexpressing cells, the majority of microtubules was restricted to the central region bounded by circumferential actin and concentrated phospho-MLC (Fig. 5h, j). With acute blebbistatin treatment, circumferential actin cables were lost and microtubules extended to the periphery, although they seemed disorganized (Fig. 5k). This suggests that rapid radial flow of actin driven by uncoupled myosin II contractility could affect microtubule organization, leading to the distinct cell morphology and vesicle distribution in adhesion-defective cells.

Consistent with previous studies ${ }^{7}$, we found that talin depletion caused loss of focal adhesions and cell rounding at later times, although it did not block initial cell spreading on fibronectin or fibronectin-dependent SFK activation. The FERM domain in the talin head has been shown to activate integrins ${ }^{4,11}$, as does the FERM domain in the kindlin family of proteins ${ }^{26}$. As expected, expression of talin1H domain in talin-deficient cells restored integrin activation and moderately increased cell adhesion to substrates. However, only full-length talin1, with both integrin-activating and actin-binding capacity, was able to restore normal actin cytoarchitecture and microtubule organization, focal adhesion assembly, and the adhesion-dependent signalling. Thus, our studies indicate that initial cell spreading and adhesion formation are separable processes, and talin 1 and 2 are involved in the primary mechanical link between 
ligand-bound integrins and the cytoskeleton, which is necessary for focal adhesion formation and substrate traction.

We did not observe any significant delay in initial cell spreading on fibronectin with talin depletion. Interestingly, initiation of spreading in both control and talin-deficient cells seems to be triggered by a fibronectin-integrin interaction, which can be blocked by soluble ligandmimetic peptide or bovine serum albumin on the surface (Supplementary Information, Fig. S2). Further, it has been shown that the delay before cells spread increases with decreasing fibronectin concentration ${ }^{27}$. Thus, we suggest that talin is not involved in the signalling process that initiates spreading through integrin binding to fibronectin. SFK activity seems to be crucial in early cell spreading ${ }^{17,18}$ and talin depletion did not affect the increase in SFK activity during spreading, whereas acute treatment with the SFK inhibitor PP2 severely interfered with the spreading of talin deficient cells (data not shown). Our observation is consistent with the hypothesis that SFK activation and initial spreading are linked events.

In fibroblasts, initiation of isotropic spreading is followed by a myosin II-independent continuous protrusion phase without prominent adhesion formation ${ }^{22,27}$. Protrusions stimulated by growth factors also proceed without adherence to substrate ${ }^{28}$. These observations suggest that actin polymerization and edge protrusion at early times do not require the engagement of talin or ECM-cytoskeleton linkage, which explains the transient spreading we observed in talin-deficient cells. Focal adhesion formation correlates with increased myosin II activity and transition to a phase of periodic contraction in fibroblasts ${ }^{22}$. Increased actomyosin contraction in the absence of talin and mature focal adhesions may finally result in rounding of adhesion-defective cells (Supplementary Information, Fig. S6a, b). Previous studies have considered a clutch that links the contractile actin network with the matrix-bound integrins ${ }^{29}$, 30. Clearly, talin is a logical candidate for the clutch and our results here show that talin is necessary for the myosin-dependent organization of actin and microtubule networks. By binding to both matrix-bound integrins and the actin cytoskeleton, talin provides a mechanical linkage that enables force generation on matrix and force-dependent activation events, as well as consolidation of adhesive linkages.

We suggest a model for the early behaviour of cell spreading and adhesion, in which the initial step involves activation of SFK-linked signalling pathways and actin polymerization by the ligand-bound integrins. After initial spreading, actomyosin contraction is activated and in the absence of full-length talin, the actin assembled in the periphery is condensed towards the central cytoplasm, holding in microtubules and cytoplasmic vesicles. The talin head activates integrin binding to the matrix but only weak forces are generated. In the presence of full-length talin, the contraction of actin filaments now pulls on liganded integrins and causes the assembly of focal adhesions and activation of force-dependent signalling. Further, coupling of actomyosin contraction to matrix contacts slows actin rearward movement, favouring spreading of microtubules. Thus, talin provides a mechanical linkage between integrins and actin filaments in cell spreading, leading to cell adhesion and substrate traction.

\section{METHODS}

\section{Cell culture}

The talin1 $^{-1-}$ fibroblast cell line dj26.28 (ref. ${ }^{7}$ ) was maintained in DMEM/F-12 medium supplemented with $15 \%$ (vol/vol) fetal bovine serum.

\section{Constructs and transfection}

A talin2 siRNA construct was generated using BD RNAi-ready pSIREN-retroQ vector according to manufacturer's instructions (BD Biosciences). Talin2 siRNA insertion: 5'- 
GATCCGAAGTCAGTATTACGTTGTTCTCAAGAGAAACAACGTAATACTGACTTC TTTTTTTCTAGAG-3'. The talin2 siRNA insertion sequence was mutated to create the control siRNA insertion sequence: 5'-

GATCCGAAGTGAGTATATCGTTGTTCTCAAGAGAAACAACGATATACTCACTTC TTTTTTTCTAGAG- ${ }^{\prime}$. All constructs were authenticated by sequencing. GFP-talin1 (mouse), GFP-talin1H (amino acids 1-435), GFP-talin1R (amino acids 434-2541) expression constructs were generated by Neil Bate (University of Leicester, UK). The GFP sequence was replaced with monomeric-DsRed to create DsRed-talin1H. Talin $1^{\mathrm{ABS}}$ lacking the C-terminal actin-binding domain (amino acids 1-2229) was from A.J. Woods (University of Leicester). $\alpha$-actinin-EGFP was from C.A. Otey (University of North Carolina, Chapel Hill). DNA constructs were transfected into talin $^{-/-}$cells using the Amaxa Nucleofector System (Lonza). About 5-10 $\mu \mathrm{g}$ of DNA was used per reaction $\left(10^{6}\right.$ cells). Efficiency, confirmed with a GFP marker, was greater than $85 \%$ after 3.5 days.

\section{Coverslip-coating, live-imaging and TIRF microscopy}

Silanized coverslips or culture dishes were coated with human plasma fibronectin $(10 \mu \mathrm{g}$ $\mathrm{ml}^{-1}$; Roche) overnight at $4{ }^{\circ} \mathrm{C}$. For live-imaging of cell spreading, cells transfected with the indicated construct were trypsinized and resuspended in culture medium for $45 \mathrm{~min}$ at $37^{\circ} \mathrm{C}$ for recovery. Cells were then sealed in a live-imaging chamber with the bottom coverslip coated with fibronectin, and mounted onto a motorized $37^{\circ} \mathrm{C}$ stage. DIC and TIRF images were taken on an Olympus BX50 fluorescence microscope with a $\times 60$, N.A. 1.45 objective.

\section{Chemicals, antibodies and immunofluorescence microscopy staining}

Blebbistatin and PP2 were from Calbiochem. GRGDTP peptide was from Sigma. Monoclonal talin antibody (8d4, WB 1:800 and IF 1:400), anti-vinculin (hVIN-1, WB 1:3000 and IF 1:500) and anti-Mena antibody (clone 21, IF 1:200) were from Sigma. Talin2-specific antibody was a gift from Dr Pietro De Camilli (Yale University). Anti-myosin IIA antibody was from Abcam (WB 1:1000). Total $\beta 1$ integrin antibody (M-106, WB 1:500) and GAPDH antibody (sc-32233, WB 1:1500) were from Santa Cruz. $\beta$-tubulin antibody (3f3, WB:1:3000 and IF 1:1000) was from Seven Hills Bioreagents. Paxillin (IF 1:500) and FAK (WB 1:500) antibodies were from BD Transduction. Anti-Src antibody (mAb327, WB 1:100) was from Calbiochem. Antiphospho-FAK antibody (pY397, WB 1:500 and IF 1:400) was from Biosource. Anti-phosphoSrc (pY416, WB 1:500), anti-phospho-MLC antibodies (Thr18/Ser19, IF 1:50) were from Cell Signaling. Monoclonal activated $\beta 1$ antibody (9EG7, IF 1:100) was from BD Pharmingen. Rhodamine-phalloidin and secondary antibodies were from Molecular Probes. For immunofluorescence microscopy, cells were fixed with $4 \%$ paraformaldehyde and permeablized with $0.1 \%$ Tritron X-100 or methanol. Primary antibody labelling was performed at $4{ }^{\circ} \mathrm{C}$ overnight and secondary antibody labelling was performed at room temperature for 45 min. Phalloidin labelling was performed together with the secondary antibody labelling. Confocal images were taken on an Olympus Fluoview FV500 laser-scanning confocal microscope with Argon $488 \mathrm{~nm}$, HeNe-G $543 \mathrm{~nm}$ and HeNe-R $633 \mathrm{~nm}$ beams. Immunofluorescence microscopy images were reconstructed from confocal $z$-slices.

\section{Western blot and quantification}

Cells in culture were rinsed once with PBS and lysed directly in RIPA buffer. For time-lapse western blotting, transfected cells were trypsinized and incubated in suspension for $45 \mathrm{~min}$ before being plated on fibronectin-coated culture dishes. At the indicated times, cells were washed once with PBS and lysed in RIPA. All cell lysates were combined with $5 \times$ loading buffer and boiled before loading onto 4-20\% gradient duramide gels (Cambrex). Protein was then transferred to Optitran reinforced nitrocellulose membrane (Whatman). The membrane was blocked with 5\% dry milk-TBST, and incubated with primary antibody overnight at $4{ }^{\circ}$ 
C. The membrane was then incubated for $1 \mathrm{~h}$ at room temperature with anti-mouse or antirabbit-HRP (Jackson Laboratories). The signal was detected with ECL western blotting detecting reagents (Amersham Biosciences) on Kodak BioMax XAR film. Signal quantification was performed with NIH ImageJ.

\section{Wrinkle gel preparation and traction force observation}

710 fluid (Dow Corning) was plasma-treated to catalyse polymerization to produce the solid surface. The surface was further UV-treated to partially de-polymerize the polymer to achieve the desirable rigidity. The wrinkle gel was coated with fibronectin $\left(10 \mu \mathrm{g} \mathrm{ml}^{-1}\right)$ for $2 \mathrm{~h}$ at 37 ${ }^{\circ} \mathrm{C}$ before performing the cell spreading assay. DIC images were taken with a $\times 40$ N.A. 0.4 objective.

\section{Supplementary Material}

Refer to Web version on PubMed Central for supplementary material.

\section{Acknowledgments}

We thank P. De Camilli for talin2-specific antibody, N. Bate for various talin1 constructs and A.J. Woods for the talin1 ABS construct. We thank N. Gauthier, T. Perez and O. Rossier for helpful discussions, and the continuing help from all members of the Sheetz laboratory. Work in the laboratory of M.P.S. was supported by the National Institutes of Health through the NIH Roadmap for Medical Research (PN2 EY016586) and X.Z. was supported by GM-003267. Work in the D.R.C. laboratory was funded by the Wellcome Trust.

\section{References}

1. Sheetz MP, Felsenfeld D, Galbraith CG, Choquet D. Cell migration as a five-step cycle. Biochem Soc Symp 1999;65:233-243. [PubMed: 10320942]

2. DeMali KA, Wennerberg K, Burridge K. Integrin signaling to the actin cytoskeleton. Curr Opin Cell Biol 2003;15:572-582. [PubMed: 14519392]

3. Shattil SJ. Integrins and Src: dynamic duo of adhesion signaling. Trends Cell Biol 2005;15:399-403. [PubMed: 16005629]

4. Critchley DR, Gingras AR. Talin at a glance. J Cell Sci 2008;121:1345-1347. [PubMed: 18434644]

5. Jiang G, et al. Two-piconewton slip bond between fibronectin and the cytoskeleton depends on talin. Nature 2003;424:334-337. [PubMed: 12867986]

6. Calderwood DA, et al. The Talin head domain binds to integrin $\beta$ subunit cytoplasmic tails and regulates integrin activation. J Biol Chem 1999;274:28071-28074. [PubMed: 10497155]

7. Priddle $\mathrm{H}$, et al. Disruption of the talin gene compromises focal adhesion assembly in undifferentiated but not differentiated embryonic stem cells. J Cell Biol 1998;142:1121-1133. [PubMed: 9722622]

8. Monkley SJ, Pritchard CA, Critchley DR. Analysis of the mammalian talin2 gene TLN2. Biochem Biophys Res Commun 2001;286:880-885. [PubMed: 11527381]

9. Small JV, Rottner K, Kaverina I, Anderson KI. Assembling an actin cytoskeleton for cell attachment and movement. Biochim Biophys Acta 1998;1404:271-281. [PubMed: 9739149]

10. Bear JE, et al. Antagonism between Ena/VASP proteins and actin filament capping regulates fibroblast motility. Cell 2002;109:509-521. [PubMed: 12086607]

11. Tadokoro $\mathrm{S}$, et al. Talin binding to integrin beta tails: a final common step in integrin activation. Science 2003;302:103-106. [PubMed: 14526080]

12. Tanentzapf G, Brown NH. An interaction between integrin and the talin FERM domain mediates integrin activation but not linkage to the cytoskeleton. Nature Cell Biol 2006;8:601-606. [PubMed: 16648844]

13. Moes M, et al. The integrin binding site 2 (IBS2) in the talin rod domain is essential for linking integrin $\beta$ subunits to the cytoskeleton. J Biol Chem 2007;282:17280-17288. [PubMed: 17430904] 
14. Lenter M, et al. A monoclonal antibody against an activation epitope on mouse integrin chain beta 1 blocks adhesion of lymphocytes to the endothelial integrin $\alpha 6$ 31. Proc Natl Acad Sci USA 1993;90:9051-9055. [PubMed: 7692444]

15. Galbraith CG, Yamada KM, Galbraith JA. Polymerizing actin fibers position integrins primed to probe for adhesion sites. Science 2007;315:992-995. [PubMed: 17303755]

16. Schlaepfer DD, Hauck CR, Sieg DJ. Signaling through focal adhesion kinase. Prog Biophys Mol Biol 1999;71:435-478. [PubMed: 10354709]

17. von Wichert G, et al. RPTP- $\alpha$ acts as a transducer of mechanical force on $\alpha 5 / \beta 3$-integrin-cytoskeleton linkages. J Cell Biol 2003;161:143-153. [PubMed: 12682088]

18. Di Florio A, et al. Src family kinase activity regulates adhesion, spreading and migration of pancreatic endocrine tumour cells. Endocr Relat Cancer 2007;14:111-124. [PubMed: 17395980]

19. Borowsky ML, Hynes RO. Layilin, a novel talin-binding transmembrane protein homologous with C-type lectins, is localized in membrane ruffles. J Cell Biol 1998;143:429-442. [PubMed: 9786953]

20. Sawada Y, Sheetz MP. Force transduction by Triton cytoskeletons. J Cell Biol 2002;156:609-625. [PubMed: 11839769]

21. Bershadsky AD, et al. Assembly and mechanosensory function of focal adhesions: experiments and models. Eur J Cell Biol 2006;85:165-173. [PubMed: 16360240]

22. Giannone G, et al. Periodic lamellipodial contractions correlate with rearward actin waves. Cell 2004;116:431-443. [PubMed: 15016377]

23. Hotulainen $P$, Lappalainen P. Stress fibers are generated by two distinct actin assembly mechanisms in motile cells. J Cell Biol 2006;173:383-394. [PubMed: 16651381]

24. Burton K, Park JH, Taylor DL. Keratocytes generate traction forces in two phases. Mol Biol Cell 1999;10:3745-3765. [PubMed: 10564269]

25. Burnette DT, et al. Filopodial actin bundles are not necessary for microtubule advance into the peripheral domain of Aplysia neuronal growth cones. Nature Cell Biol 2007;9:1360-1369. [PubMed: 18026092]

26. Moser M, et al. Kindlin-3 is essential for integrin activation and platelet aggregation. Nature Med 2008;14:325-330. [PubMed: 18278053]

27. Dubin-Thaler BJ, Giannone G, Dobereiner HG, Sheetz MP. Nanometer analysis of cell spreading on matrix-coated surfaces reveals two distinct cell states and STEPs. Biophys J 2004;86:1794-1806. [PubMed: 14990505]

28. Bailly M, Condeelis JS, Segall JE. Chemoattractant-induced lamellipod extension. Microsc Res Tech 1998;43:433-443. [PubMed: 9858340]

29. Ponti A, et al. Two distinct actin networks drive the protrusion of migrating cells. Science 2004;305:1782-1786. [PubMed: 15375270]

30. Hu K, et al. Differential transmission of actin motion within focal adhesions. Science 2007;315:111115. [PubMed: 17204653] 
a



C



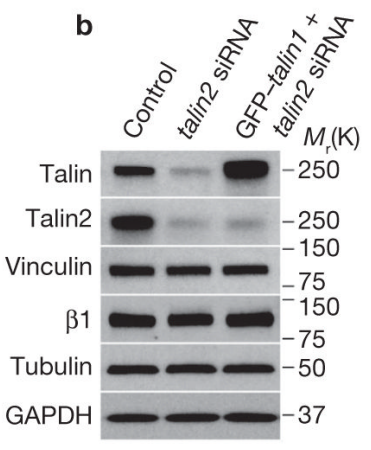



f



Figure 1.

Talin2 depletion in talin ${ }^{-/-}$cells does not affect initial spreading on fibronectin but causes cell rounding. (a) GFP and talin2 siRNA co-transfected cells rounded 90 min after plating on fibronectin-coated coverslip. GFP and control siRNA or GFP-talin1 and talin2 siRNA cotransfected cells showed a normal elongated fibroblast-like morphology. (b) The decrease in talin2 levels ranged from 48-68\%, depending on the transfection efficiency of talin2 siRNA. (c, d) Time-lapse sequential images of the $\operatorname{talin} 1^{-/-}$control cell (c, Supplementary Information, Movie 1) and the talin-deficient cell (d, Supplementary Information, Movie 2) spreading on fibronectin. (e) Summary of early spreading behaviours of control and talin-deficient cells. (f) Time lapse between initiation of spreading and complete rounding up of talin-deficient cells. Scale bars are $50 \mu \mathrm{m}(\mathbf{a}), 10 \mu \mathrm{m}(\mathbf{c}, \mathbf{d})$. 




Talin2


Figure 2.

Spread talin-deficient cells show defects in focal adhesion formation and adhesion to substrates. (a-h) Talin-deficient cells spread without assembling focal adhesions. Control siRNA (a, $, \mathbf{c}, \mathbf{e}, \mathbf{g})$ and talin 2 siRNA $(\mathbf{b}, \mathbf{d}, \mathbf{f}, \mathbf{h})$ transfected cells were plated on fibronectin for 20 min before fixation and were stained for the indicated proteins and F-actin. (i, j) Talindeficient cells spread with defective adhesion to the substrate. GFP and control siRNA (i) or GFP and talin2 siRNA (j, Supplementary Information, Movie 3) co-transfected cells were plated on fibronectin. DIC and TIRF images of live cells were taken at $10 \mathrm{~s}$ per frame. Scale bars are $5 \mu \mathrm{m}(\mathbf{c})$ and $10 \mu \mathrm{m}(\mathbf{a}, \mathbf{e}, \mathbf{g}, \mathbf{i})$ 






c



d



e


Figure 3.

Talin1 head activates $\beta 1$ integrin but does not rescue focal adhesion formation. (a) The spread phase on fibronectin was prolonged in cells expressing the talin 1 head (talin $1 \mathrm{H})$ but not the talin $1 \operatorname{rod}\left(\right.$ talin1R). Talin $1^{-1-}$ cells co-transfected with GFP-talin1H and talin2 siRNA or with GFP-talin $1 R$ and talin2 siRNA were plated on fibronectin for 90 min before fixation. (b) Summary of early spreading behaviour of talin $1 \mathrm{H}$-expressing cells. (c) Sequential time-lapse images of the talin1H-expressing cell spreading on fibronectin (Supplementary Information, Movie 4). (d, e) Focal adhesion formation was defective in talin $1 \mathrm{H}$-expressing cells. $90 \mathrm{~min}$ after plating, cells were fixed and stained for vinculin $(\mathbf{d})$ and paxillin $(\mathbf{e})$. (f-h) Talin, through its head domain, activated and colocalized with $\beta 1$ integrin at the leading edge of spread cells. 
GFP-talin1-restored cells (f), GFP-talin1H-expressing cells (g) and talin-deficient cells (h) were plated on fibronectin for $20 \mathrm{~min}, 90 \mathrm{~min}$ and $20 \mathrm{~min}$, respectively before fixation. Scale bars are $50 \mu \mathrm{m}(\mathbf{a}), 5 \mu \mathrm{m}(\mathbf{e})$ and $10 \mu \mathrm{m}(\mathbf{c}, \mathbf{d}, \mathbf{f}-\mathbf{h})$. 
。

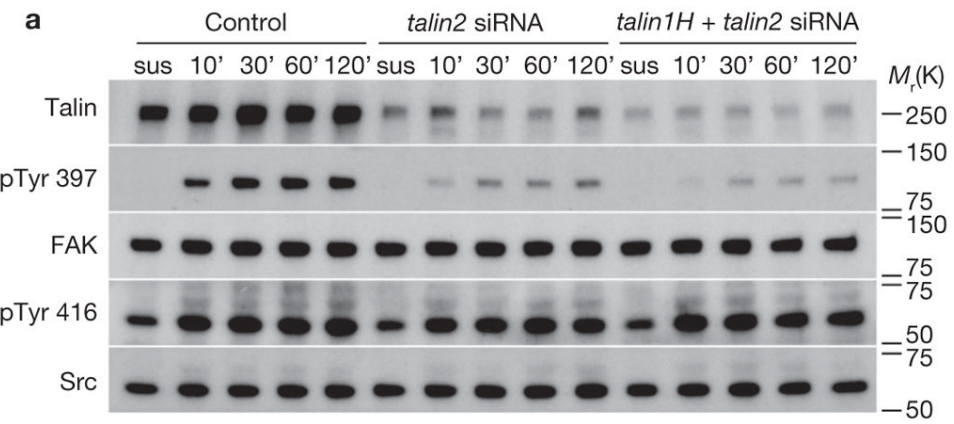

b pTyr 397: FAK on FN

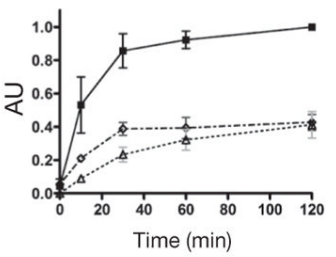

C pTyr 416: Src increase on FN

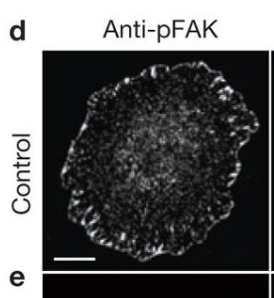

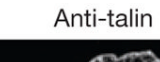







$\rightarrow$ Control

$\rightarrow$ talin2 siRNA

$\rightarrow$ talin1 $H+$ talin2 siRNA

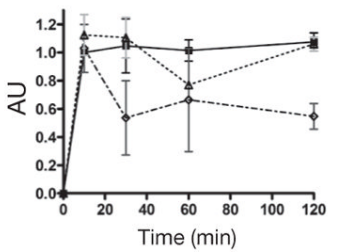

me (min)

Anti-pFAK

GFP F-actin



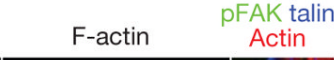





Figure 4.

Talin depletion impairs FAK-Tyr 397 phosphorylation upon adhesion to fibronectin. (a)

Western blots showed defects in adhesion-dependent FAK-Tyr 397 phosphorylation in talindeficient cells. Control, talin2 siRNA, and talin1H and talin2 siRNA co-transfected cells were plated on fibronectin-coated culture dishes for the indicated time before lysis. (b) Profile of FAK-Tyr 397 phosphorylation levels with adhesion to fibronectin. pTyr 397: FAK ratio of control cells at $120 \mathrm{~min}$ was set at 1 arbitrary unit (AU) and the values of other time points were adjusted proportionally. Data are mean \pm s.e.m., $n=3$. (c) Activation profile of SFK with adhesion to fibronectin. pTyr 416: Src ratio increase of $10 \mathrm{~min}$ above the suspension sample in control cells was set at $1 \mathrm{AU}$ and other time points were adjusted proportionally. Data are mean \pm s.e.m., $n=3$. (d-f) Immunostaining for phospho-FAK (pTyr 397) in control (d), talin2deficient (e) and talin1H-expressing cells (f). Cells were fixed $20 \mathrm{~min}(\mathbf{d}, \mathbf{e})$ and $90 \mathrm{~min}(\mathbf{f})$ after plating. The pTyr 397 signal was lost in talin2-deficient and talin1H-expressing cells. Scale bars are $10 \mu \mathrm{m}$. 
a



c



b



Width of

fast rearward flow region

d



Width of fast rearward flow region







Figure 5.

Talin couples actomyosin contractility to the substrate and facilitates the distribution of microtubules. (a-d) Cells without full-length talin could not confine fast $\alpha$-actinin rearward flow to the leading edge. Talin $1^{-1-}$ cells co-transfected with $\alpha$-actinin-GFP and control siRNA (a, Supplementary Information, Movie 6) or $\alpha$-actinin-GFP, DsRed-talin1H and talin2 siRNA (c, Supplementary Information, Movie 7) were plated on fibronectin. DIC, TIRF or epifluorescence (epi) sequential images were taken at $10 \mathrm{~s}$ per frame. Kymographs (b, d) were generated from Supplementary Information, Movies 6 and 7, respectively. There was partial loss of the TIRF signal in $\mathbf{d}$ caused by ventral membrane detachment. Regions with fast rearward flow and the flow rate were determined by kymographs (averaged from three measurements for each cell). Scale bars are $5 \mu \mathrm{m}$ (a, c) and 1 min (horizontal), $2 \mu \mathrm{m}$ (vertical) $(\mathbf{b}, \mathbf{d})$. (e,f) Blebbistatin treatment $(\mathrm{Bb}, 50 \mu \mathrm{M})$ slowed the rearward flow in talin $1 \mathrm{H}$-expressing cells, as revealed by the kymograph of DIC (f, Supplementary Information, Movie 8). Cell flattening was observed immediately after drug perfusion. Images before and $2.5 \mathrm{~min}$ after treatment were shown (e). Scale bars are $10 \mu \mathrm{m}(\mathbf{e}), 1 \mathrm{~min}$ and $5 \mu \mathrm{m}(\mathbf{f})$. $(\mathbf{g}, \mathbf{h})$ Coupling of actomyosin contractility to the substrate was affected in the absence of full-length talin. GFPtalin1 and talin2 siRNA or GFP-talin1H and talin2 siRNA co-transfected cells were plated on fibronectin and stained for phospho-MLC. (i-k) Actomyosin activity restricted microtubules in talin1H-expressing cells. Talin1H-expressing cells $(\mathbf{k})$ were plated in the same 
way as talin1-restored (i) and talin1H-expressing (j) cells, but were treated with $50 \mu \mathrm{M}$ blebbistatin for $2.5 \mathrm{~min}$ before fixation. Scale bars are $10 \mu \mathrm{m}(\mathbf{g}-\mathbf{k})$. 